\title{
The Effective Application of Mother-of-pearl Lacquerware Technology in Modern Product Design
}

\author{
JunGuo ZHAO
}

\author{
Suan Sunandha Rajabhat University \\ *348300571@qq.com
}

\begin{abstract}
Firstly, this paper analyzes the development of lacquerware and mother-of-pearl lacquerware, including the application and development of lacquer modeling and mother-of-pearl lacquerware. Then, it elaborates the application of mother-of-pearl lacquerware technology in modern product design, including the application of mother-of-pearl lacquerware technology in accessories design, mother-of-pearl lacquerware technology in packaging design, motherof-pearl lacquerware technology in edible utensils design and mother-of-pearl lacquerware technology in public art design. Finally, it analyzes the inheritance value of mother-of-pearl lacquerware in modern product design, including the spiritual inheritance value and practical inheritance value of mother-of-pearl lacquerware. In order to make the traditional mother-of-pearl lacquerware technology be combined with modern technology, inheritance and development.
\end{abstract}

Keywords: Mother-of-pearl Lacquerware, Technology, Modern, Product Design

\section{INTRODUCTION}

Lacquerware technology has lasted for thousands of years since ancient times. In the development history of thousands of years, the most famous one is "bodiless lacquerware", which is produced in Yichun, Jiangxi. During the Ming and Qing Dynasties, Beijing cloisonne, Jingdezhen porcelain and Yichun bodiless lacquerware were known as the three treasures of China. With the development and change of the times, western culture invaded China in the late Qing Dynasty. In people's sight, the traditional lacquerware technology was gradually replaced by western culture, and the development of lacquerware culture was also restricted. Until the founding of New China, lacquerware technology was inherited and protected, which made the machine technology break the original confinement, and lacquerware and mother-of-pearl got a good development. The Shang Dynasty was the earliest era when the two were integrated with each other. The mother-of-pearl was inlaid on lacquerware by carving and cutting, thus obtaining the new handicraft of embedded mother-of-pearl. Mother-of-pearl lacquerware technology was first used in the daily necessities design of furniture design. With the continuous progress and development of lacquerware technology, mother-of-pearl lacquerware technology is not only limited to the design of daily necessities machines, but also widely used in other product design and artistic decoration. In the design of modern products, the technology of mother-of-pearl lacquerware is also integrated into it.

\section{DEVELOPMENT OF LACQUERWARE AND MOTHER-OF-PEARL LACQUERWARE}

\subsection{Application and Development of Lacquerware Modeling}

Lacquerware first appeared in the Neolithic period, when lacquerware was only used in daily life. In other words, it is a technological product derived from low social productivity. Bionic is the original lacquerware modeling, and there is no beauty, just to meet the needs. One of the more famous lacquer bowl it was unearthed in the Hemudu period, vermilion raw paint attached to its interior, only covering a thin layer, its shape is relatively symmetrical, also more rules, oval melon diamond shape, in this period, practical is still the main function of lacquer[1]. In this stage, lacquerware was created by simulating the shapes of animals and plants in nature. In this period, people had only one practical requirement for lacquerware, and sketched lacquerware 
according to the requirements. Until the pre-Qin period, bronzes influenced the creation and modeling of lacquerware. At this stage, the colors of lacquerware gradually became more solemn and rich. The development of culture also drives the development of machines. People began to apply lacquerware to sacrificial vessels and musical instruments in the Spring and Autumn Period. Lacquerware modeling developed to the Qin and Han Dynasties, and the craft and modeling of lacquerware received more attention, not only limited to the original practicality. In the Eastern Han Dynasty, Yichun bodiless lacquerware, which is well known to modern people, appeared. Among them, the most well-known one is the wine utensil in Mawangdui-the lacquer ear cup box, which is shaped like an ear, hence its name[2]. Compared with other lacquerware, the lacquer ear cup is not only beautiful but also practical. The wisdom of the ancients can also be reflected from the lacquer ear cup, and the lacquer ear cup does not occupy much space. The progress and development of the times also led to the progress and development of lacquer ware technology. Lacquerware developed to the Sui and Tang Dynasties, the traditional shape of lacquerware could not meet the needs of the audience, and people's aesthetics gradually changed, requiring more exquisite lacquerware with more special shape. At this time, inlays were started on lacquerware, and the materials used for inlaying were snail or gold and silver. Lacquerware in this period was unique in shape and gorgeous and beautiful. The mature state of lacquerware technology appeared in the Ming and Qing Dynasties, when the shape of lacquerware was simpler and more novel than before. The shape of petals gradually appears on the shape of lacquer ware, and the inlays on the surface are still retained[3].

\subsection{Application and Development of Mother- of-pearl Lacquerware}

The earliest lacquerware was used to hold food. By the time of Liangzhu culture, edible lacquerware could not meet the demand, and household lacquerware, including case and barrel, was gradually derived. At this stage, lacquerware was more widely used than before. According to the archaeological research in China, we can know that the decoration of mother-of-pearl lacquerware technology appeared as early as the Western Zhou Dynasty. Although there are not many unearthed cultural relics, it is enough to prove that the art of mother-of-pearl lacquerware technology is recognized by people. According to historical records and archaeological discoveries, it was in Yuan and Song Dynasty that the snail embedding machine was widely popularized among the people. In the modeling design and manufacturing process of lacquerware, the motherof-pearl lacquerware technology was gradually applied. Until the Ming Dynasty, the lacquerware for household decoration was developed and applied. At this time, the mother-of-pearl lacquerware technology greatly improved the aesthetics of the lacquerware for household decoration. In this period, cabinets, tables and boxes were made with lacquerware, and the screwinlaid technique was also applied to inlay them[4]. This also marks the mutual integration of the screw-inlaid craft and lacquer ware, which is inseparable from the screw-inlaid lacquer ware in all aspects of people's daily life.

\section{APPLICATION OF MOTHER-OF- PEARL LACQUERWARE TECHNOLOGY IN MODERN PRODUCT DESIGN}

\subsection{Application of Mother-of-pearl Lacquerware Technology in the Field of Accessories Design}

It is an aesthetic instinct for people to decorate themselves. Since ancient times, the pursuit of beauty has continued. At first, when people decorate themselves, they will choose animal feathers. With the development and continuous progress of society, people's pursuit of beauty has changed. In the design of modern products, the field of accessories design is constantly changing with the change of audience aesthetics. Nowadays, the audience of products is more modern women, who are sensitive to the change of color and have higher requirements for lacquerware technology. The mother-of-pearl lacquerware technology is more widely used in the design of modern product accessories. For example, the red paint comb, which is deeply loved by the Miao nationality, also applied the inlay technology of snail in product design. The comb is more exquisite and gorgeous, and is liked by consumers. In modern product design, the mother-ofpearl lacquerware technology is widely used in cigarette cases, mirrors, watches, bracelets and necklaces. The design of these accessories is more beautiful and artistic due to the auxiliary design of mother-of-pearl lacquerware technology. Due to the collision and effective combination of Chinese traditional culture, traditional crafts and modern product design, the exquisite degree and artistic sense of accessories have been improved, which makes them popular among the public[5].

\subsection{Application of Mother-of-pearl Lacquerware Technology in the Field of Packaging Design}

In modern product design, more and more attention has been paid to packaging design. Packaging design not only needs to meet the practicality, but also needs to have aesthetic features to ensure that the designed packaging can be recognized and liked by the audience and attract the attention of the audience. The 
development of packaging design changes with the change of people's aesthetics, and the application range of mother-of-pearl lacquerware technology in packaging design is further expanded. Of course, mother-of-pearl lacquerware technology can not only make the packaging more beautiful, but also prolong the service life of products to a certain extent by applying lacquerware materials in packaging design because lacquer ware materials are more damage-proof and firm. For example, in the design process of wine bottle, lacquerware technology can make the bottle cover more beautiful, and the application of mother-of-pearl lacquerware technology and lacquer carving technology in the packaging design of wine bottle can make the wine bottle more beautiful and has collection value. Carving the cutting materials with embedded furnace technology can carve patterns with Chinese characteristics, such as plum, orchid, bamboo and chrysanthemum. The design of modern wine bottle products incorporates elements of Chinese style, and at the same time, the mother-of-pearl lacquerware technology is used to assist the design. This is the collision between modern design and traditional technology, which can improve the value of wine bottle, effectively improve the shelf life of wine, promote the brand value and promote the economic benefits. This packaging design that meets the needs of modern people and conforms to modern aesthetics is worth popularizing and continuing[6].

\subsection{Application of Mother-of-pearl Lacquerware Technology in the Field of Edible Utensils Design}

In the design of practical appliance products, lacquerware is widely used. Not only is machine technology widely used in the design of practical tools in China, but also lacquerware is widely used in the design of practical tools in other countries. For example, Japan's Tsugaru chopsticks, etc. In modern people's life, practical utensils are not only food needs, but also the exquisite workmanship and aesthetic degree of edible utensils are important factors that determine whether the audience buys them or not. In the design process of practical appliances, the auxiliary design of the motherof-pearl lacquerware technology is also important. In the design of practical appliances, the technology-aided design of mother-of-pearl lacquerware can not only make practical appliances more beautiful, but also effectively inherit and carry forward the technology of mother-of-pearl lacquerware. In the modern tea culture and food culture, the mother-of-pearl lacquerware technology is constantly infiltrated, which makes the diversity of lacquerware reflected. However, with the development and progress of society, lacquerware has not keep pace with the times, which leads to the motherof-pearl lacquerware technology is not widely used in practical vessel design. Mother-of-pearl embedded materials can be used in edible appliances design in a larger range, which can effectively broaden the route of edible appliance design. The audience's needs for edible appliances can be met with the assistance of mother-ofpearl embedded materials, making daily necessities more delicate and beautiful.

\subsection{Application of Mother-of-pearl Lacquerware Technology in the Field of Public Art Design}

The field of public art design covers a lot of contents, including items displayed indoors, objects and objects in the outdoor environment. Interior wall design is an important part of interior display design, such as hotel meeting rooms and hotels. The interior wall design is more important, and it can be applied to the interior wall design with mother-of-pearl lacquerware technology, which can greatly improve the aesthetics and practicality of interior wall design. The mother-ofpearl embedded material is hard and not easy to be corroded, which can prolong the service life of interior wall, and has both practicality and aesthetics. Motherof-pearl embedded materials can also be used in home decoration design in interior decoration design, such as screen partition or furniture design. Using modern technology to design mother-of-pearl embedded materials while retaining traditional mother-of-pearl lacquerware technology is the collision between information technology and traditional technology, which can add color to interior decoration. The outdoor environment design in the field of public art design needs to choose raw materials that are corrosionresistant and not easy to deform, and the mother-ofpearl embedded materials can just meet the demand[7]. Considering the overall decorative effect and structure of outdoor space, glass porcelain, plastic products and wood products that are easy to break and wear can't be used alone in outdoor environment. These materials can be added with mother-of-pearl embedded materials, which can realize the interactive use of various materials, improve the aesthetics of outdoor space to a certain extent, and at the same time play a certain role in protecting raw materials. The luster of mother-of-pearl lacquerware is relatively beautiful, and it is more modern. Not only mother-of-pearl lacquerware, but also other lacquerware are widely used in public design fields, such as street lamps and public seats with relatively large demand in public design, etc. They all paint on the basis of raw materials, which prolongs the service life and improves the aesthetic degree. Modern craft modeling combined with traditional lacquerware art is more conducive to the development of public art design[8]. 


\section{THE INHERITANCE VALUE OF MOTHER-OF-PEARL LACQUERWARE IN MODERN PRODUCT DESIGN}

\subsection{The Spiritual Inheritance Value of Mother- of-pearl Lacquerware}

Mother-of-pearl lacquerware technology in modern product design has the value of spiritual inheritance. In product design, designers pass on their own design ideas. The application of mother-of-pearl lacquerware technology in present products can make people know something about Chinese traditional culture and traditional lacquerware technology, and its spiritual connotation can be passed on to more people, which makes the audience and designers have emotional resonance, and is also conducive to the inheritance and development of lacquer craftsman spirit. Embedding mother-of-pearl lacquerware into modern product design can make the designed products have a sense of science and technology while being integrated into traditional culture, and thus the traditional culture and technology are recognized by more people. The lacquer civilization has lasted from the Stone Age to the present, and it should be recognized and valued by modern people[9]. The application of mother-of-pearl lacquerware in handicrafts, daily necessities and even decorations also conveys the spirit of lacquer artisans, making the inheritance of lacquer spirit more valuable. Consumers can feel the thoughts, spirits and technological skills conveyed by designers through products.

\subsection{The Practical Inheritance Value of Mother-of-pearl Lacquerware}

Aesthetics and practicality are very important for modern product design. Lacquerware, one of the products of aesthetic and practical combination, occupies an important position in modern product design. Mother-of-pearl lacquerware has practical inheritance value in modern product design. In people's daily life, lacquerware is one of the traditional utensils, and its modeling features are distinctive. Generally speaking, the boxes of lacquerware are mostly round or square, which is more convenient for daily storage. For example, the jewelry boxes that girls often use are made of wood materials and mother-of-pearl embedded materials, which are both beautiful and practical. This lacquerware design makes it convenient and orderly for the audience to receive in the use process, and it can also save the use space to a certain extent. This lacquerware design process is widely used in modern packaging design and modern product design. In the modern home design, there are many applications of mother-of-pearl lacquerware, such as common hanging pictures and ornaments. Painting needs to enrich the colors of the home space, and at the same time, it can enhance the users' sense of happiness. The gorgeous and shining mother-of-pearl embedded materials can attract the audience's attention and promote the purchase desire. Traditional ornaments only have ornamental value. Modern product design pays more attention to the combination of practicality and ornamental value. The storage box made by mother-of-pearl lacquerware technology can meet the basic performance of ornaments for viewing, and can also store some smaller items. Compared with traditional ornaments, the practicality is higher. This also indicates the practical inheritance value of mother-of-pearl lacquerware in modern product design[10].

\section{CONCLUSION}

From the Neolithic Age to the modern society, lacquerware technology has been inherited and developed through countless times of changes, among which the mother-of-pearl lacquerware technology is widely used in modern product design. The mother-ofpearl lacquerware technology makes the product of the combination of traditional culture and ingenuity need to be inherited and continued. Modern product design meets the needs of modern audiences, and it also needs designers to pay attention to guiding audiences' aesthetics. Beauty should not be defined. Now technology is combined with traditional acquerware technology, and modern craft is combined with motherof-pearl lacquerware materials. The products designed in this way are beautiful and irreplaceable. This is the craft of our nation and the treasure of our traditional culture.

\section{ACKNOWLEDGMENTS}

I deeply appreciate Asst. Prof. Dr. Pisit Puntien of Suan Sunandha Rajabhat University for his guidance and encouragement to this paper.

\section{REFERENCES}

[1] Li Li.Night Dialogue Stars - - Comment on Li Aizhen Mother-of-pearl Lacquerware Art[J].Art Observation,2021(02):128-129.

[2] Ji Fang, Pei Hanqiu.The Skill and Aesthetic Feeling of Mother-of-pearl Lacquerware[J].Popular Literature and Art,2020(11):82-83.

[3] Li Aizhen.Design and Manufacture of Jishan Mother-of-pearl Lacquerware[J].Journal Of Chinese Lacquer,2020,39(01):31-33.

[4] Li Yujin. Aesthetic Characteristics of Mother-ofpearl Lacquerware and Jewelry Design Practice[D].China University of Geosciences(Beijing),2019. 
[5] Xu Hualei, Li Lu, Wei Yuying.The Effective Application of Mother-of-pearl Lacquerware Technology in Modern Product Design[J].Appreciation,2019(08):1-2.

[6] Lian Xiaohui.Analysis on the characteristics and development of Jishan Mother-of-pearl Schema[J].Art Science And Technology,2017,30(05):67+100.

[7] Lu Hangyu. Manufacturing Technique of Flat Grinding Mother-of-pearl Lacquerware [N]. China Cultural Heritage News,2017-09-15(007).

[8] Xing Na.The Decorative Performance of Motherof-pearl Inlaid in Lacquerware[J].Journal of Chinese Lacquer,2017,36(02):11-15.

[9] Guo Caiping. Study on the Traditional Technology of Mother-of-pearl Lacquerware in Jishan[D].Shanxi University,2017.

[10] Ma Guitang, Ma Jing.The Art of Colored Shell Inlay - - Jishan Technology of Mother-of-pearl Lacquer[J].Journal

Of

Chinese Lacquer,2010,29(02):49-52. 